\title{
A Case of Giant Macular Hole Progression after Rupture of a Giant Retinal Pigment Epithelial Detachment
}

\author{
Kosuke Nagaoka ${ }^{a}$ Satoru Inoda ${ }^{a}$ Hidenori Takahashi ${ }^{a, c}$ Yusuke Arai ${ }^{a}$ \\ Yuji Inoue $^{a}$ Yuichi Takizawa ${ }^{b}$ Shinkin Fujioka ${ }^{c}$ Hidetoshi Kawashima $^{a}$ \\ aDepartment of Ophthalmology, Jichi Medical University, Shimotsuke, Japan; bHigashi \\ Totsuka Denen Eye Clinic, Yokohama, Japan; 'Totsuka Eye Clinic, Yokohama, Japan
}

\section{Keywords}

Macular hole · Pigment epithelial detachment · Retinal pigment epithelial tear

\begin{abstract}
We report a case of giant macular hole progression after rupture of giant retinal pigment epithelial detachment (PED). The patient was a 91-year-old man who had a giant PED in the left eye. He had bilateral hypermetropia (+2.00 dpt), and he had developed posterior vitreous detachment. The PED was 5,800 $\mu \mathrm{m}$ in diameter and $800 \mu \mathrm{m}$ in height and ruptured during follow-up. A macular hole was formed, with a diameter of $400 \mu \mathrm{m}$, and the height of the PED had reduced to $360 \mu \mathrm{m}$. After 5 months, the macular hole expanded up to a diameter of $600 \mu \mathrm{m}$. Therefore, some cases of giant PED may lead to macular hole.

\section{Introduction}

Pigment epithelial detachment (PED) may occur under various conditions, with or without neovascularization, usually associated with age-related macular degeneration. Although 
PED sometimes heals spontaneously, a large PED expands gradually in the majority of cases and may rupture, causing pigment epithelial defect within a few years [1].

Idiopathic macular hole $(\mathrm{MH})$ is caused by progression of vitreomacular traction from posterior vitreous detachment (PVD) due to aging when strong vitreous adherence to the fovea exists. Although cases of $\mathrm{MH}$ were originally reported in the setting of trauma, other causes or mechanisms such as retinal vitreous surgery or diabetic edema have been reported [2-5]. Here, we present a novel MH formation that occurred after rupture of a giant PED.

\section{Case Report}

The patient was a 91-year-old man with a chief complaint of distorted vision in the left eye. On initial examination at Higashi Totsuka Denen Eye Clinic (by Y.T.), best-corrected visual acuity (BCVA) was 20/25 for oculus dexter (OD) and 20/50 for oculus sinister (OS); refractive error was $+3.25 /-2.50 \times 100^{\circ}(\mathrm{OD})$ and $+4.50 /-2.00 \times 70^{\circ}(\mathrm{OS})$, and intraocular pressure (IOP) was $12 \mathrm{~mm} \mathrm{Hg}$ for both OD and OS. PVD had already developed in the left eye. Numerous drusen were present in both eyes. PED was noted, with a diameter of 5,800 $\mu \mathrm{m}$ and height of $800 \mu \mathrm{m}$ in the left eye (Fig. 1a). Ophthalmoscopy showed no findings suggestive of CNV such as subretinal hemorrhage, elevated gray or orange lesions, or a notch of PED. Optical coherence tomography revealed that there was no type $2 \mathrm{CNV}$, double-layer sign, or polypoidal lesion under the pigment epithelium. In addition, no CNV was observed during the clinical course.

After 3 months, he was referred to a second clinic because of worsening of symptoms and examined there (Totsuka Eye Clinic by H.T. and S.F.). At that time, BCVA was 20/20 OD and 20/125 OS, IOP was $12 \mathrm{~mm} \mathrm{Hg}$ for OD and $11 \mathrm{~mm} \mathrm{Hg}$ for OS; there was an MH and a PED, with the height reduced to $360 \mu \mathrm{m}$ in his left eye (Fig. 1b).

Considering his age and systemic condition, we did not perform MH surgery because it was difficult for him to lie face down for the period required for the procedure. So, we continued with monthly examinations using a spectral domain optical coherence tomography system (RS-3000 Lite, Nidek Co., Ltd., Aichi, Japan) and a nonmydriatic ultra-widefield fundus camera (Daytona, Optos Inc., Marlborough, MA, USA). After a month, retinal pigment epithelium (RPE) had developed a tear and had contracted downward, creating a large circular pigment epithelium defect. The MH progressed to retinal detachment (Fig. 2).

After 6 months, fibrovascular proliferative tissue had spread to the subretinal space and the MH was adherent to Bruch's membrane. The MH retinal detachment disappeared but the diameter of the MH increased to $600 \mu \mathrm{m}$ (Fig. 3). After 11 months, there was no particular change from the previous examination. BCVA was 20/320 OS. The size of the hole remained $600 \mu \mathrm{m}$. One year after the initial presentation, the patient died.

\section{Discussion/Conclusion}

Some reports have described the processes of MH formation in PED. Cazabon [6] stated that in some cases of MH formation there may be a causative link with PED. Storch and Hoerauf reported that as PED increases, the retina might become stretched, and lateral extension force could create an MH [7].

In the present case, it is difficult to confirm the possible mechanism of $\mathrm{MH}$ formation because the patient had hypermetropia with PVD. If the MH was formed when the PED increased, 
the retina should have been thinned. In the present case, there were no signs of retinal thinning at the time the first OCT was taken, and the PED is thought to be chronic due to the absence of CNV. It is unlikely that the PED significantly expanded and caused retinal thinning in the 3 months after the first OCT, so the MH was probably formed after the rupture rather than when the PED expanded. The lower height of PED is thought to be related to the beginning of RPE rupture, because the large RPE tear occurred after the PED reduction. The MH was confirmed before the contraction of the pigmented epithelium, and so there is a possibility that a different force than has been reported might be responsible for forming the $\mathrm{MH}$, such as ejection of subretinal fluid to the vitreous side early after the rupture began. In fact, $\mathrm{MH}$ formation after spontaneous extraction of subfoveal perfluorocarbon liquid has been reported [8].

In this case, the general mechanism of idiopathic $\mathrm{MH}$ formation by vitreomacular traction is not the cause because of the hypermetropia with PVD. We speculated that the MH occurred during the expansion of the PED or after the PED rupture due to a distinct unique force, such as a laterally extending force in the direction of the retina. Thereafter, the RPE shrunk to become a mass, the extension force increased, and the MH expanded.

\section{Statement of Ethics}

This research complies with the relevant guidelines for human studies and was conducted ethically in accordance with the World Medical Association Declaration of Helsinki. The study protocol was approved by the IRB of Jichi Medical University. Patient consent to review his medical records was not required by the IRB, because the analyzed data does not contain any personally identifiable data and the patient was already deceased.

\section{Disclosure Statement}

Kosuke Nagaoka, Satoru Inoda, Yusuke Arai, Yuichi Takizawa, and Shinkin Fujioka have no conflict of interests to declare. Hidenori Takahashi received grants and personal fees from Novartis Pharmaceutical and personal fees from Senju Pharmaceutical, Bayer Pharmaceutical, Santen Pharmaceutical, Pfizer Inc., and Kowa Pharmaceutical unrelated to this report. Hidenori Takahashi has a pending patent and is a co-founder of DeepEyeVision LLC. Yusuke Arai received grants from Novartis Pharmaceutical unrelated to this report. Yuji Inoue received personal fees from Bayer Pharmaceutical, Novartis Pharmaceutical, and Santen Pharmaceutical unrelated to this report. Hidetoshi Kawashima received personal fees from Santen Pharmaceutical, Mitsubishi-Tanabe Pharmaceutical, and Senju Pharmaceutical unrelated to this report.

\section{Funding Sources}

This research received no specific grant from any funding agency in the public, commercial, or not-for-profit sectors. 


\section{Case Reports in Ophthalmology}

\section{Author Contributions}

K.N. designed and conducted this study, performed data acquisition and analysis, and wrote the text of the main manuscript. H.T. designed and conducted this study. H.K. supported and supervised the study. All authors reviewed and approved the manuscript.

\section{References}

1 Laatikainen L. Rupture of retinal pigment epithelial detachment in senile macular disease. Acta Ophthalmol (Copenh). 1983 Feb;61(1):1-8.

2 Ho AC, Guyer DR, Fine SL. Macular hole. Surv Ophthalmol. 1998 Mar-Apr;42(5):393-416.

3 Noyes HD. Detachment of Retina with Laceration at Macula. Trans Am Ophthalmol Soc. 1871;1(8):128-9.

4 Amemiya T, Yoshida H. Macular hole in diabetic maculopathy. Ophthalmologica. 1978;177(4):188-91.

5 Lee SH, Park KH, Kim JH, Heo JW, Yu HG, Yu YS, et al. Secondary macular hole formation after vitrectomy. Retina. 2010 Jul-Aug;30(7):1072-7.

6 Cazabon S. Full-thickness macular hole formation associated with pigment epithelial detachment: link or coincidence? Int Ophthalmol. 2010 Dec;30(6):739-42.

7 Storch MW, Hoerauf H. Case report of a secondary macular hole closure after intravitreal bevacizumab therapy in a patient with retinal pigment epithelial detachment. Indian J Ophthalmol. 2017 Jul;65(7):632-3.

8 Tanabu R, Suzuki Y, Suzuki K, Takahashi S, Nakazawa M, Mizutani H. Macular hole caused by retained subfoveal perfluorocarbon that subsequently closed after its spontaneous resolution: A case report. Ophthalmol Ther. 2017 Dec;6(2):381-4.
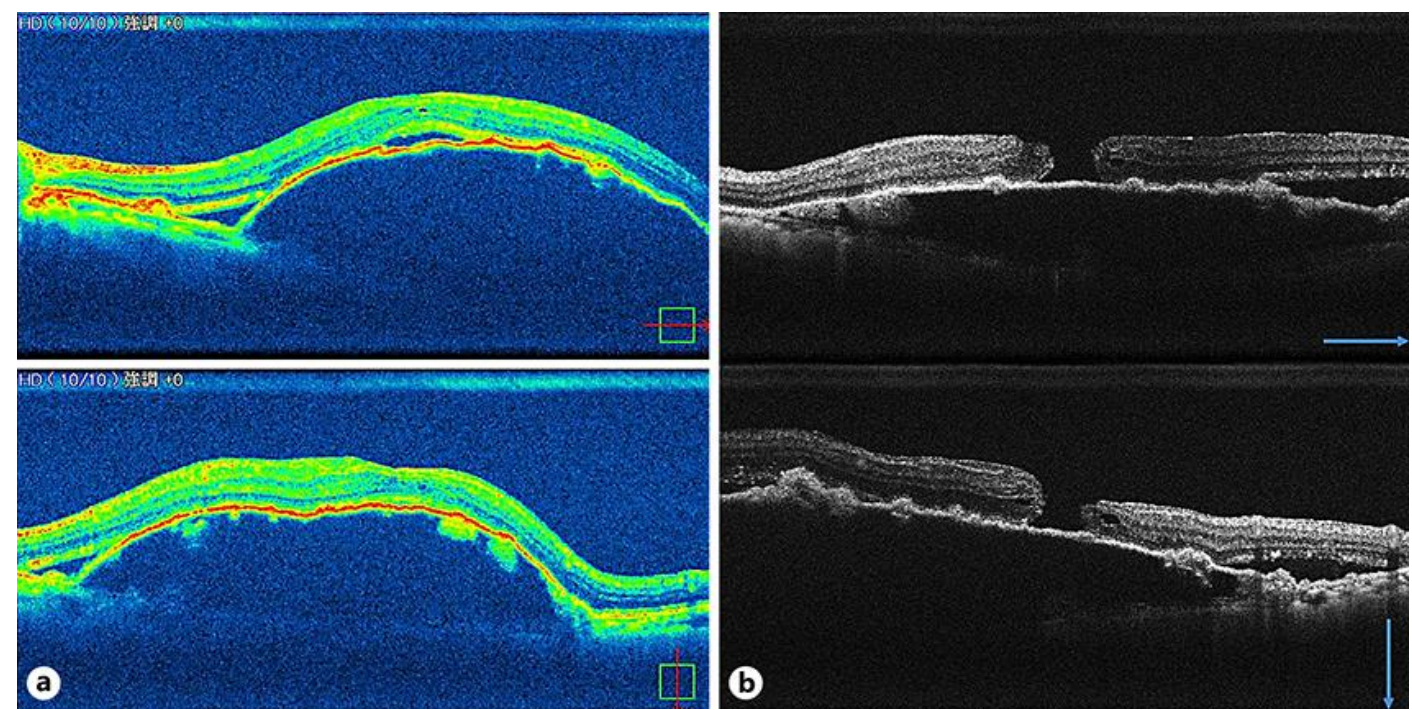

Fig. 1. Optical coherence tomography (OCT) horizontal and vertical images. a OCT at the first examination at a local eye clinic. Pigment epithelial detachment is seen in the left eye (diameter, 5,800 $\mu \mathrm{m}$; height, 800 $\mu \mathrm{m})$. b Initial optical coherence tomography images at the second local clinic, 3 months later. A macular hole is seen with pigment epithelial detachment (height, $360 \mu \mathrm{m}$ ) and serous retinal detachment. 


\section{Case Reports in Ophthalmology}
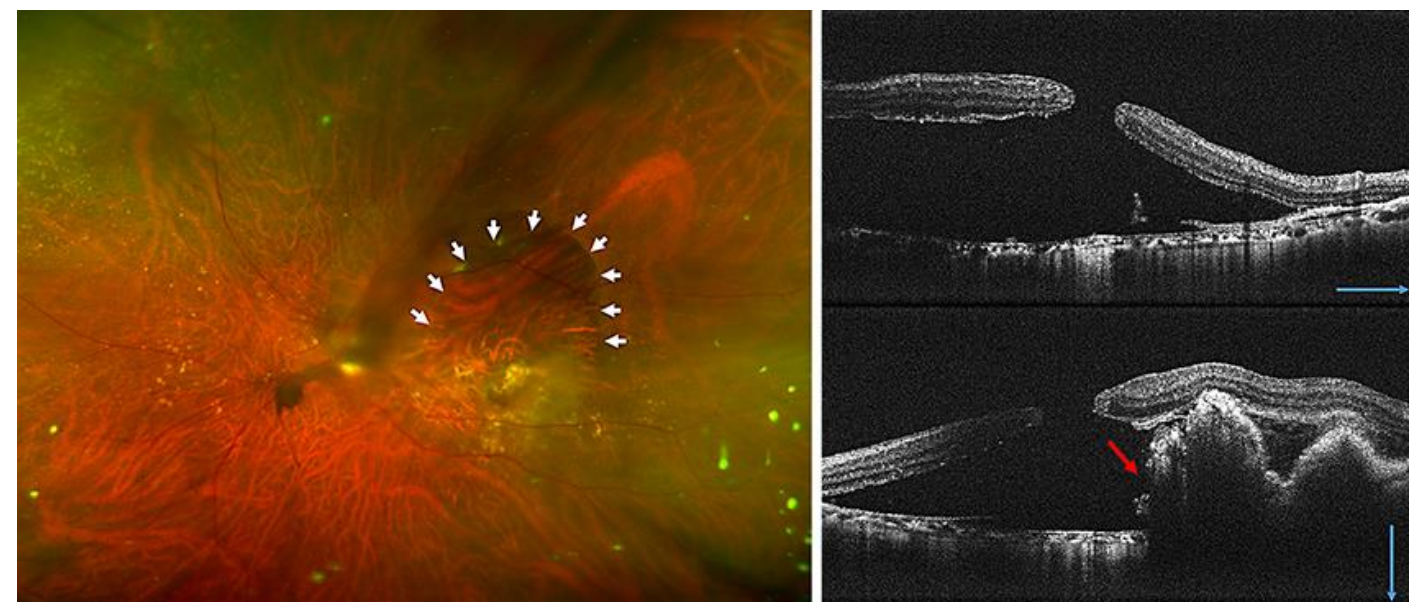

Fig. 2. Optos ${ }^{\circledR}$ ultra-widefield color fundus image and optical coherence tomography images after 1 month. White arrows show the edge of pigment epithelial detachment; red arrow shows retinal pigment epithelium that has contracted downwards.
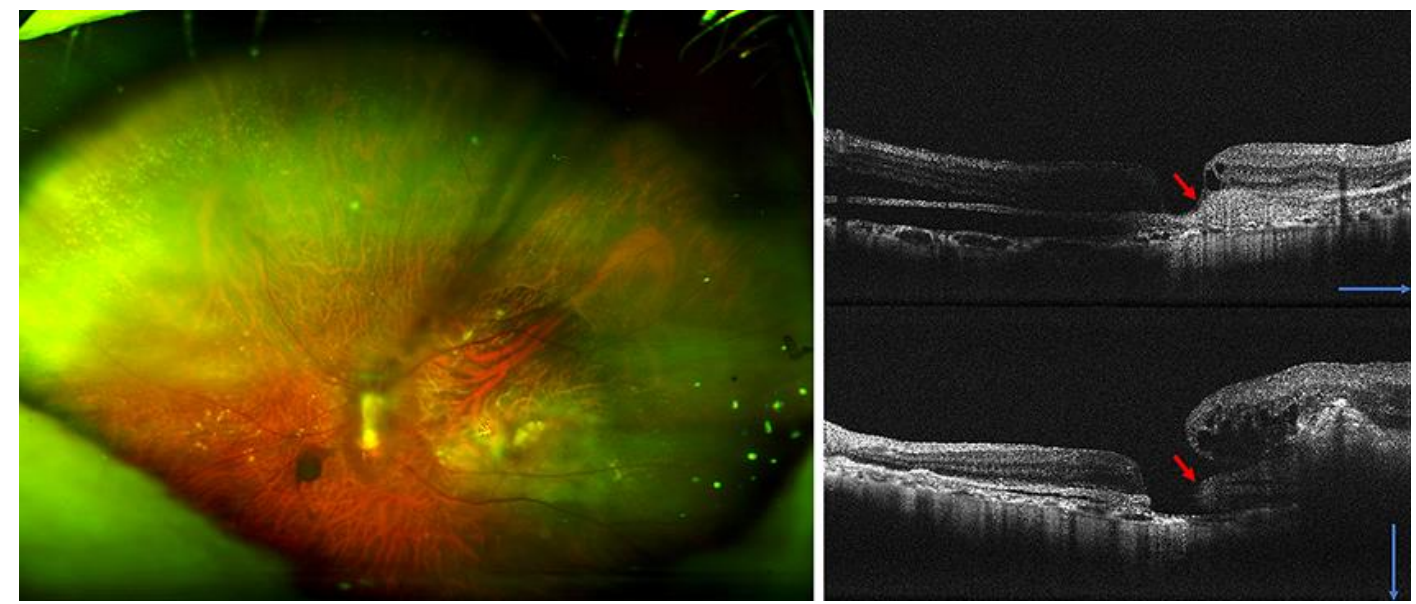

Fig. 3. Images after 6 months. Red arrows show fibrovascular proliferative tissue from the retinal pigment epithelium mass. The macular hole retinal detachment has disappeared, and the macular hole diameter has expanded to $600 \mu \mathrm{m}$. No features of choroidal neovascularization were observed such as subretinal hemorrhage, branching vascular network, or elevated gray or orange lesions. 